\title{
Antibody Response against SARS-CoV-2 Following ChAdOx1(Astezeneca AZD 1222) Vaccine in Health Care Workers in a Tertiary Care Hospital in India
}

Felice FAIZAL ${ }^{1}$, Rajneesh JOSHI², R CHATTERJEE ${ }^{1}$, Ankur AHUJA ${ }^{1}$

\begin{abstract}
BACKGROUND: The ChAdOx1 nCoV-19 vaccine against COVID-19 is a two-dose vaccine spread 3 to 4 weeks apart. This study aims to ascertain the antibody response to each dose with respect to age, previous infection status etc. METHODS: Baseline total COVID-19 antibody level was ascertained using Siemens SARS-CoV-2 Total Antibody assay in consenting health care workers before the first dose of vaccination. Adverse effects were noted in each individual and were monitored weekly for the total antibody titre following both doses. Descriptive statistical tests were used to analyse the changes in antibodies levels weekly after both doses. Association of previous COVID infection and age with antibody levels was assessed. RESULTS: Median (range) of age of the 30 study participants was 31.5 years. $23 \%$ of the participants had a history of previous COVID-19 infection. Mild adverse events following immunisation were reported by $87 \%$ participants after first dose whereas only in $7 \%$ after second dose. Median baseline antibody titres were significantly higher among those with previous COVID infection as compared to previously uninfected individuals. Antibody titres increased consistently after first dose and showed a declining trend following the second dose in all participants and showed no significant association with previous COVID-19 infection or age. CONCLUSIONS: Antibody titre response was similar amongst the various age groups. Higher response in the previously infected individuals following first dose may make them ideal candidates for a single dose vaccine regimen. Individuals showing lower levels of neutralising antibodies can be ideal candidates for a booster dose.
\end{abstract}

Keywords: COVID-19 vaccine, ChAdOx1 nCoV-19, influenza vaccines, vaccination and immunity.

Department of Pathology, Army Medical Corps, India ${ }^{2}$ Department of Preventive and Social Medicine, Army Medical Corps, India
Corresponding author:

Felice Faizal, A 201, Kailash towers, IGNOU road, 110030, New Delhi, India.

E-mail: flcfzl@gmail.com 


\section{INTRODUCTION}

As a global response to the COVID-19 pandemic, several vaccines against the SARS-Cov2 virus have been simultaneously developed by various agencies and are forming the backbone of humanities fight against the disease through targeted and mass vaccinations ${ }^{1,2}$.

Developed by Oxford University and AstraZeneca, the COVID-19 vaccine, codenamed AZD1222 or ChAdOx1 nCoV-19 vaccine, is sold under the brand names Covishield and Vaxzevria. The vaccine uses the modified chimpanzee adenovirus $\mathrm{ChAdOx} 1$ as a viral vector and is administered intramuscularly in two doses spread 3 to 4 weeks apart.

ChAdOx1 $\mathrm{nCoV}-19$ is a replication-deficient simian adenoviral vector that expresses the full-length SARS-CoV-2 spike protein. In preclinical studies, either a single dose or two doses of

ChAdOx1 nCoV-19 vaccination prevented SARS$\mathrm{CoV}-2$-mediated pneumonia in rhesus macaques ${ }^{3}$.

Further clinical studies have shown that even a single dose of this vaccine is producing a strong antibody response in more than 80 percent of the human recipients ${ }^{4}$.

Even though antibody response and $\mathrm{T}$ cell response are the main arms of the adaptive immune response following vaccination against viral pathogens, the mechanisms and duration of protective immunity in COVID19 remain to be elucidated.

Earlier this year, the health care workers (HCWs) at our facility were administered both the doses of this vaccine at an interval of 4 to 6 weeks. This study aims to ascertain the safety and the antibody response to each dose and study its pattern with respect to age, previous infection status and duration between doses.

\section{MATERIAL AND METHODS}

A pilot study group of $30 \mathrm{HCW}$ s was identified between the ages of 25 to 50 and their previous medical history was noted. After obtaining informed consent to participate in this study, a baseline total COVID19 antibody (Ig G + Ig M) level was ascertained using Siemens automated biochemistry analyser parameter SARS-CoV-2 Total Antibody assay (COV2T) which is a chemiluminescent assay detecting the concentration of total antibodies to SARS-CoV-2 in human serum and plasma and an absorbance value of greater than 1000 QUAL units is considered as positive. The absorbance is directly proportional to the concentration of antibodies in the serum/plasma.

Following the first dose of vaccination, adverse effects were noted in each individual and all the study subjects were monitored serially on a weekly basis for the total antibody titre using the same test as before.

This was followed by the second dose of vaccination at an interval of 4 to 8 weeks and the gap between the doses for each individual, adverse effects after the second dose and weekly total antibody titres were recorded as before.

\section{Statistical analysis}

Data was summarised by median, inter-quartile range and proportions. Antibody levels in two groups were compared by Mann Whitney test. Linear mixed effect models were used for analyzing the changes in antibodies levels over time after first and second vaccine dose. Association of previous COVID infection and age with antibody levels was also assessed. Variables with $\mathrm{p}<0.1$ in univariable analysis were included for multivariable analysis. $\mathrm{R}$ software ver 3.6.3 was used for statistical analysis and nlme package was used for linear mixed effect modelling. $p$ value of less than 0.05 was statistically significant.

\section{Ethical issues}

Approval of the study was obtained from Institutional Ethics Committee. Informed consent of all study participants was taken.

Informed consent of all study participants was taken.

\section{RESULTS}

A total of $30 \mathrm{HCW}$ participated in this study, out of whom only $2(7 \%)$ were female. Median (range) of age of study participants was $31.5(25-50)$ years. No study participant had any comorbidity. Seven (23\%) of the participants had a history of previous COVID-19 infection, of which 2 recovered from mild, 4 from moderate and 1 from severe disease. Median (range) gap between previous infection and first dose of vaccination was $153(132-214)$ days for these seven individuals. Mild adverse events following immunisation were reported by 26 (87\%) study participants after first dose - most common being myalgia (16 HCW, 53\%) and fever (13 HCW, 43\%). Only 2 (7\%) HCW reported mild fever and myalgia after second dose. 


\section{Baseline titres}

Median (IQR) baseline antibody titres before first dose of vaccine were 437 (234 -826) IU/L. These titres were significantly higher among those with previous COVID infection as compared to individuals with no documented COVID infection (5000 IU/L vs 292 IU/L). Baseline antibody titres of $6(85.7 \%)$ of previously infected individuals were $>1000$ Qual units.

\section{Change in antibody titres after first dose}

Antibody titres increased consistently after first dose in all participants till 3 weeks after first dose (Figure 1).
Median (IQR) antibody titres after first, second and third week following prime dose of vaccine were 7046 (5429 - 14106), 29835 (16295 - 45252) and 45093 (25438 - 51765) respectively. Though antibody titres were higher among previously infected HCW, the difference was not statistically significant except after first week (Table 1). Average increase in antibody titres (after adjusting for previous COVID infection) was around 13000 QUAL units every week for the first three weeks. No significant association of increase in titres after first dose was observed with history of previous COVID-19 infection or age (Table 2 \& Figure 2).

Table 1. Week wise Antibody Values with Inter Quartile Range

\begin{tabular}{|c|c|c|c|c|}
\hline & $\begin{array}{l}\text { Total } \\
(n=30)\end{array}$ & $\begin{array}{l}\text { No previous COVID infection group } \\
\qquad(\mathrm{n}=23)\end{array}$ & $\begin{array}{l}\text { Previous COVID infection group } \\
\qquad(\mathrm{n}=7)\end{array}$ & $P$ value \\
\hline Baseline & $\begin{array}{c}437 \\
(233.8-826)\end{array}$ & $\begin{array}{c}292 \\
(197-356)\end{array}$ & $\begin{array}{c}5000 \\
(1759-10610)\end{array}$ & 0.0001 \\
\hline$D+7$ days & $\begin{array}{c}7046 \\
(5429-14106)\end{array}$ & $\begin{array}{c}6555 \\
(4739-12367)\end{array}$ & $\begin{array}{c}26783 \\
(14605-44668)\end{array}$ & 0.0077 \\
\hline$D+14$ days & $\begin{array}{c}29835 \\
(16295-45252)\end{array}$ & $\begin{array}{c}28742 \\
(16502-42399)\end{array}$ & $\begin{array}{c}45379 \\
(18688-61353)\end{array}$ & 0.3478 \\
\hline$D+21$ days & $\begin{array}{c}45093 \\
(25438-51765)\end{array}$ & $\begin{array}{c}42744 \\
(25764-51088)\end{array}$ & $\begin{array}{c}48806 \\
(24512-63499)\end{array}$ & 0.6005 \\
\hline$D+28$ days & $\begin{array}{c}15434 \\
(10511-31306)\end{array}$ & & & \\
\hline $\mathrm{DD}+7$ days & $\begin{array}{c}39609 \\
(23212-44872)\end{array}$ & $\begin{array}{c}39474 \\
(25628-44861)\end{array}$ & $\begin{array}{c}39823 \\
(28738-47011)\end{array}$ & 0.6692 \\
\hline $\mathrm{DD}+14$ days & $\begin{array}{c}29837 \\
(15400-38776)\end{array}$ & $\begin{array}{c}28843 \\
(14690-37661)\end{array}$ & $\begin{array}{c}33677 \\
(19837-42691)\end{array}$ & 0.4467 \\
\hline $\mathrm{DD}+21$ days & $\begin{array}{c}16545 \\
(8832-18712)\end{array}$ & $\begin{array}{c}16545 \\
(8832-18712)\end{array}$ & & \\
\hline $\mathrm{DD}+28$ days & $\begin{array}{c}9665 \\
(6439-12100)\end{array}$ & $\begin{array}{c}8924 \\
(6180-10733)\end{array}$ & $\begin{array}{c}19255 \\
(10735-27506)\end{array}$ & 0.0799 \\
\hline
\end{tabular}

Table 2. Linear mixed model analysis showing average difference per week following first dose of vaccination

\begin{tabular}{|l|l|l|l|l|}
\hline & \multicolumn{2}{|l|}{ Univariable Analysis } & \multicolumn{2}{l|}{ Multivariable analysis } \\
\hline & $\begin{array}{l}\text { Regression } \\
\text { Co-efficient }\end{array}$ & P value & & \\
\hline $\begin{array}{l}\text { Time } \\
\text { (Per Week) }\end{array}$ & $\begin{array}{l}13023 \\
(8722,14982)\end{array}$ & $<0.0001$ & $\begin{array}{l}13009 \\
(11050,14967)\end{array}$ & $<0.0001$ \\
\hline $\begin{array}{l}\text { Previous COVID } \\
\text { infection } \\
(\text { Yes Vs No) }\end{array}$ & $\begin{array}{l}11412 \\
(-87,22912)\end{array}$ & 0.0516 & $\begin{array}{l}11103 \\
(-241,22446)\end{array}$ & 0.0547 \\
\hline Age & $\begin{array}{l}16 \\
(-637,668)\end{array}$ & 0.9610 & - & - \\
\hline
\end{tabular}




\section{Change in antibody titres after second dose}

As depicted in Fig 1 and Table 3, average decline of 8096 units per week was observed in antibody levels after second dose of vaccine. Median (IQR) antibody titres fell from 39609 (23212 - 44872) one week after second dose to $9665(6439-12100)$ after four weeks. This declining trend was observed in individuals with previous COVID infection as well as in those with no history of previous COVID infection (Figure 3).

Table 3. Linear mixed model analysis showing average difference per week following second dose of vaccination

\begin{tabular}{|l|c|c|}
\hline & \multicolumn{2}{|c|}{ Univariable Analysis } \\
\hline & $\begin{array}{c}\text { Regression } \\
\text { Co-efficient }\end{array}$ & P value \\
\hline Time (Per Week) & $\begin{array}{c}-8096 \\
(-9543,-6649)\end{array}$ & $<0.0001$ \\
\hline $\begin{array}{l}\text { Previous COVID } \\
\text { infection }\end{array}$ & $\begin{array}{c}4157 \\
(-6485,14800)\end{array}$ & 0.4287 \\
\hline Age & $\begin{array}{c}-96 \\
(-625,433)\end{array}$ & 0.7121 \\
\hline
\end{tabular}

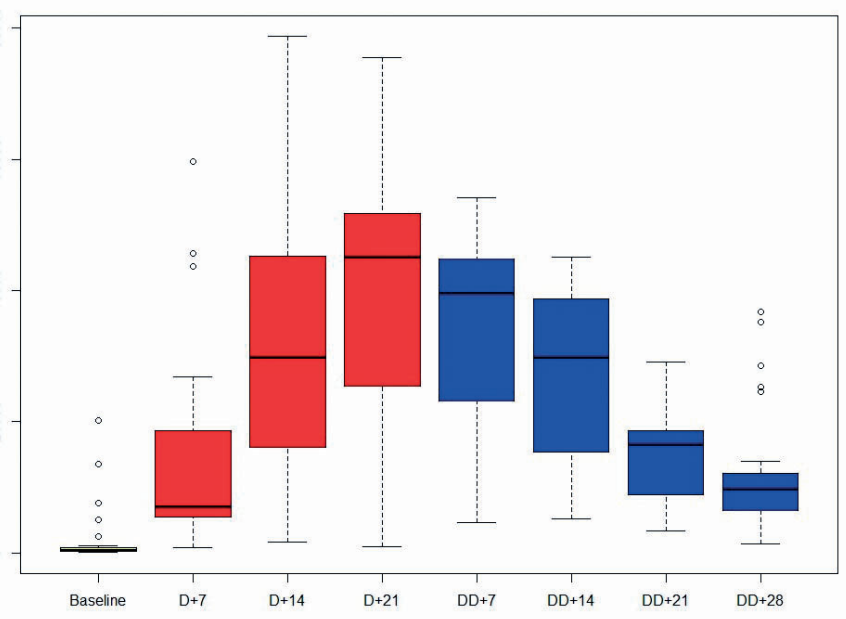

Figure 1. Box and whisker plot showing antibody titres post first dose(D) and second dose (DD).
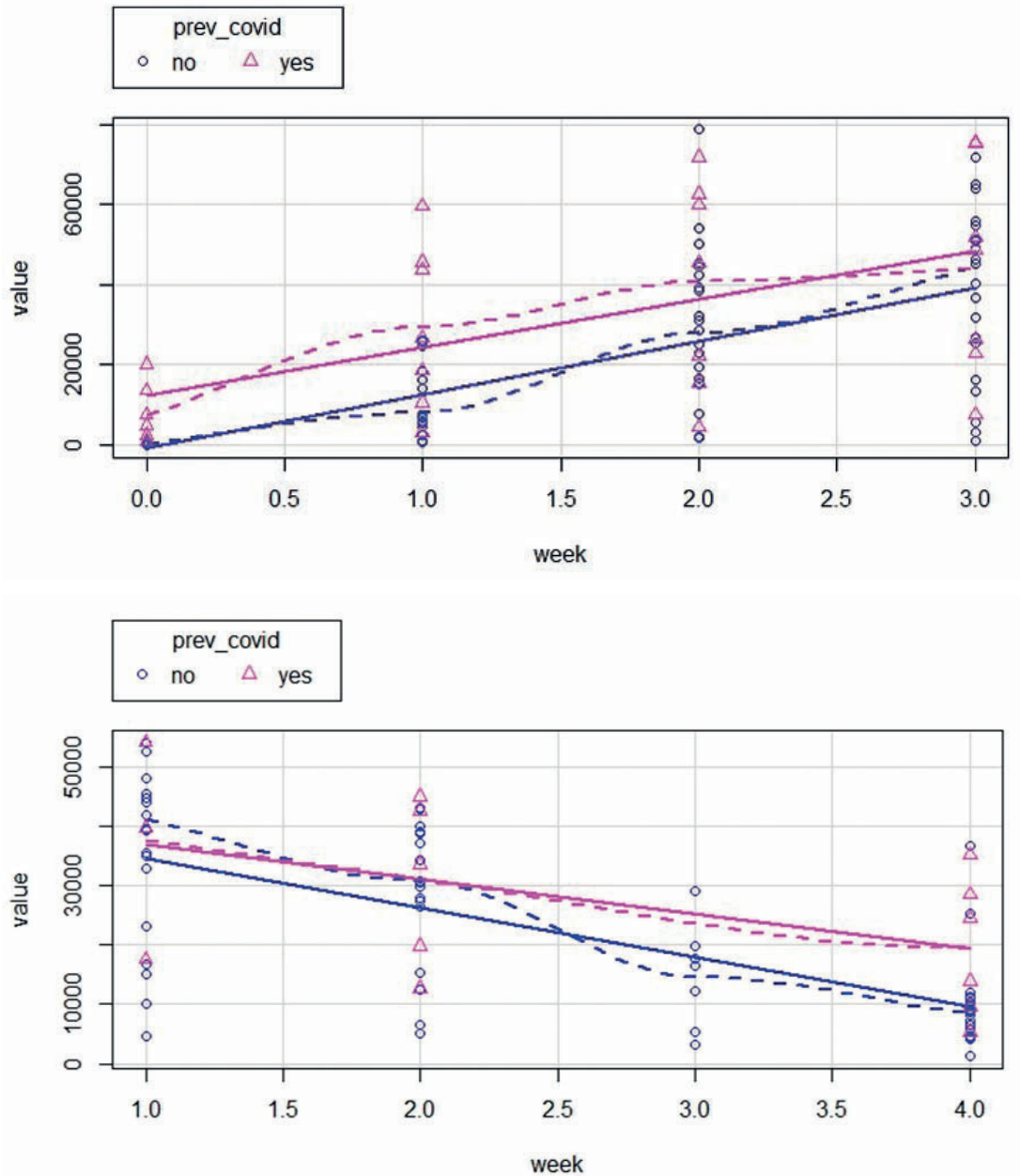

Figure 2. Linear mixed model showing a rising trend in the antibody titres during the first three weeks post first dose

Figure 3. Linear mixed model showing a decreasing trend in the antibody titres during the weeks post second dose. 


\section{DISCUSSION}

The adaptive immune response to SARS-CoV-2 virus comprises $\mathrm{T}$ and $\mathrm{B}$ cell immunity and antiviral neutralizing antibodies. The antibody-mediated immune response against the SARS-CoV-2 virus is primarily aimed at the spike glycoprotein and $\mathrm{N}$ (nucleocapsid) $\operatorname{protein}^{5,6}$.

The AstraZeneca AZD1222 vaccine is a safe double dose vaccine ideally to be administered at an interval of 4 to 6 weeks.

Mild Systemic and local side effects were noted in all previously uninfected individuals which were alleviated by single dose of NSAIDS which was similar to the findings of the phase 1 and 2 randomized control trials ${ }^{7}$.

However, amongst the previously infected and recovered individuals who received the vaccine in our study, only 57 percent exhibited mild systemic side effects like fever and myalgia. These results contrast with the observations by Menni et al who found a low adverse effect rate of 33.7 percent with the ChAdOx1 vaccine compared to the 88 percent in the phase 2 and 3 trials. They also noted that that Individuals with evidence of past SARS-CoV-2 infection were more likely to have adverse effects than those without past infection with this vaccine ${ }^{8}$.

Inspite of initial recommendations of a 4-to-6week interval between the two doses, in many regions, the second dose of the vaccine was delayed by up to 3 months to ensure greater population coverage of the first dose?

The findings of our study show definite advantage of this strategy as the rate of seroconversion following the first dose of the vaccine was greater than 90 percent suggesting adequate interim protection following a single dose.

Jeewandara et al elicited similar results in Sri Lanka where a robust rate of seroconversion was seen after the initial dose of the two-part vaccine.in addition ACE 2 blocking antibodies were formed in more than 97 percent of their naïve vaccine recipients ${ }^{10}$.

Similarly, a metaanalysis by Voysey et al demonstrated a high efficacy of a single dose of vaccine in the first 90 days with better immunogenicity with a longer duration between doses ${ }^{11}$.

This suggests that a single dose provides adequate protection for a duration for as long as 90 days, and this may be exploited to maximise the first dose coverage of the population.
It was noted that the baseline titres of all the previously COVID-19 infected individuals in the current study was significantly higher than the previously uninfected group which suggests that previous infection provides a sound immune response to the disease. However Various studies have suggested that the principle functional immunity is governed by the memory B cells and memory $\mathrm{T}$ cells and decreases over time even if it doesn't disappear ${ }^{12,13}$. Therefore vaccine should not be denied to the previously infected and recovered individuals. They may however be ideal candidates for a single dose regimen or may be placed low on the vaccination priority list as antibody response amongst the previously infected individuals following the first dose of vaccination was higher than the previously uninfected population initially which suggests a higher response to a single dose of vaccine amongst the previously infected. Like our findings, Saadat et al also observed that Health care workers with previous COVID-19 infection had higher antibody titre responses to a single dose of mRNA vaccine than those who were not previously infected ${ }^{14}$.

In our study, the antibody response increased consistently following the first dose in all the individuals. The previously infected individuals who had received the vaccine showed a greater response in terms of rising antibody titre in the initial 3 weeks, but no significant difference was seen in the response thereafter. This suggests that the antibody response following the first dose of the vaccine is as potent as previous infection in terms of seroconversion.

With respect to the age groups, no significant difference was seen in the immune response to the vaccine across various age groups. Ramasamy et al also observed that in participants who received two doses of vaccine, median anti-spike SARS-CoV-2 IgG responses were similar across the three age cohorts in their study ${ }^{15}$.

The overall trend of total antibody titres in our study shows an initial rise in the total titres followed by a steady fall in the total titres following the second dose. This corroborates the observations in the systematic review conducted by Post et al in which levels of IgM antibody peaked between 2 to 5 weeks and declined over the next 3 to 5 weeks. IgG peaked around weeks three to seven post-symptom onset then plateaued, generally persisting for at least eight weeks ${ }^{16}$.

None of the subjects included in this study contracted COVID-19 infection post vaccination till date which suggests good vaccine efficacy. This may be due 
to the limited sample size of our study as various reports of post vaccination breakthrough infection have been reported worldwide with the incidence dramatically decreasing with each passing week post vaccination ${ }^{17}$.

\section{CONCLUSION}

1. ChAdOx1 nCoV-19 Vaccine, also known as Astra Zeneca AZD1222 and Covishield is a safe and effective vaccine with a very robust rate of seroconversion even after the first dose.

2. No difference was seen in the antibody titre response amongst the various age groups.

3. The previously infected individuals mount a higher antibody response in the first few weeks following the first dose of vaccination, however no significant difference is noted thereafter between the infected and the uninfected group.

4. The previously infected group may be ideal candidates for a single dose vaccine regimen or can be placed low on the priority list.

5. Since the total titres fall over an extended period, specific tests can be done to detect neutralising antibodies and to detect the cellular response. Individuals showing lower levels of either can be ideal candidates for a booster dose.
Compliance with ethics requirements: The authors declare no conflict of interest regarding this article. The authors declare that all the procedures and experiments of this study respect the ethical standards in the Helsinki Declaration of 1975, as revised in 2008(5), as well as the national law. Informed consent was obtained from all the patients included in the study. 


\section{References}

1. Oliver SE, Gargano JW, Marin M, et al. The Advisory Committee on Immunization Practices' Interim Recommendation for Use of Pfizer-BioNTech COVID-19 Vaccine - United States, December 2020. MMWR Morb Mortal Wkly Rep 2020; 69(50): 1922-4.

2. Dagan N, Barda N, Kepten E, et al. BNT162b2 mRNA Covid-19 Vaccine in a Nationwide Mass Vaccination Setting. The New England journal of medicine 2021.

3. Van Doremalen, $\mathrm{N}$. et al. ChAdOx $1 \mathrm{nCoV}-19$ vaccine prevents SARS-CoV-2 pneumonia in rhesus macaques. Nature 586, 578$582(2020)$

4. Mahase E. Covid-19: Single dose of Pfizer or AstraZeneca vaccine produces strong antibody response in over 80s BMJ 2021; 373:n979 doi:10.1136/bmj.n979

5. Poland GA, Ovsyannikova IG, Kennedy RB. SARS-CoV-2 immunity: review and applications to phase 3 vaccine candidates. Lancet. 2020 Nov 14;396(10262):1595-1606. doi: 10.1016/S01406736(20)32137-1. Epub 2020 Oct 13. PMID: 33065034; PMCID: PMC7553736

6. Hossein Ansariniya, Seyed Mohammad Seifati, Erfan Zaker, Fateme Zare, "Comparison of Immune Response between SARS, MERS, and COVID-19 Infection, Perspective on Vaccine Design and Development", BioMed Research International, vol. 2021, Article ID 8870425, 11 pages, 2021. https://doi.org/10.1155/2021/8870425

7. Pedro M Folegatti, Katie $J$ Ewer, Parvinder $K$ Aley et al.Safety and immunogenicity of the ChAdOx $1 \mathrm{nCoV}-19$ vaccine against SARS-CoV-2: a preliminary report of a phase $1 / 2$, single- blind, randomised controlled trial, The Lancet, Volume 396, Issue 10249,2020,Pages 467- 478, ISSN 0140-6736, https://doi. org/10.1016/S0140-6736(20)31604-4

8. Menni C, Klaser K, May A, et al. Vaccine side-effects and SARSCoV-2 infection after vaccination in users of the COVID Symptom Study app in the UK: a prospective observational study. The Lancet. Infectious Diseases. 2021 Apr. DOI: 10.1016/ s1473- 3099(21)00224-3.

9. Pimenta D, Yates C, Pagel C, Gurdasani D. Delaying the second dose of covid-19 vaccines. BMJ (Clinical research ed 2021; 372: n710.

10.Chandima Jeewandara, Achala Kamaladasa, Pradeep Darshana Pushpakumara et al. Antibody and $\mathrm{T}$ cell responses to a single dose of the AZD1222/Covishield vaccine in previously SARS-CoV-2 infected and naive health care workers in Sri Lanka medRxiv 2021.04.09.21255194; doi: https://doi. org/10.1101/2021.04.09.21255194

11. Voysey M, Costa Clemens SA, Madhi SA, et al; Oxford COVID Vaccine Trial Group. Single-dose administration and the influence of the timing of the booster dose on immunogenicity and efficacy of ChAdOx1 nCoV-19 (AZD1222) vaccine: a pooled analysis of four randomised trials. Lancet. 2021 Mar 6;397(10277):881891. doi: 10.1016/S0140-6736(21)00432-3. Epub 2021 Feb 19. Erratum in: Lancet. 2021 Mar 6;397(10277):880. PMID: 33617777 PMCID: PMC7894131.
12. Hartley GE, Edwards ESJ, Aui PM, Varese N, Stojanovic S, McMahon J, Peleg AY, Boo I, Drummer HE, Hogarth PM, O'Hehir RE, van Zelm MC. Rapid generation of durable B cell memory to SARS-CoV-2 spike and nucleocapsid proteins in COVID-19 and convalescence. Sci Immunol. 2020 Dec 22;5(54):eabf8891. doi: 10.1126/sciimmunol.abf8891. PMID: 33443036; PMCID: PMC7877496.

13. Yamayoshi $S$, Yasuhara $A$, Ito $M$, Akasaka $O$, Nakamura $M$, Nakachi I, Koga M, Mitamura K, Yagi K, Maeda K, Kato H, Nojima M, Pattinson D, Ogura T, Baba R, Fujita K, Nagai H, Yamamoto S, Saito M, Adachi E, Ochi J, Hattori SI, Suzuki T, Miyazato Y, Chiba S, Okuda, Murakami J, Hamabata T, Iwatsuki-Horimoto K, Nakajima H, Mitsuya H, Omagari N, Sugaya N, Yotsuyanagi H, Kawaoka Y. Antibody titers against SARS-CoV-2 decline, but do not disappear for several months. EClinicalMedicine. 2021 Feb;32:100734. doi: 10.1016/j.eclinm.2021.100734. Epub 2021 Feb 11. PMID: 33589882; PMCID: PMC7877219.

14. Saadat S, Rikhtegaran Tehrani Z, Logue J, et al. Binding and Neutralization Antibody Titers After a Single Vaccine Dose in Health Care Workers Previously Infected With SARS-CoV-2. JAMA. 2021;325(14):1467-1469. doi:10.1001/jama.2021.3341

15. Ramasamy, Maheshi $N$ et al. "Safety and immunogenicity of ChAdOx $1 \mathrm{nCoV}$-19 vaccine administered in a prime-boost regimen in young and old adults (COV002): a single-blind, randomised, controlled, phase 2/3 trial." Lancet (London, England) vol. 396,10267 (2021): 1979-1993. doi:10.1016/S0140-6736(20)32466-1

16. Post N, Eddy D, Huntley C, van Schalkwyk MCl, Shrotri M, Leeman D, Rigby S, Williams SV, Bermingham WH, Kellam P, Maher J, Shields AM, Amirthalingam G, Peacock SJ, Ismail SA. Antibody response to SARS-CoV-2 infection in humans: A systematic review. PLoS One. 2020 Dec 31;15(12):e0244126. doi: 10.1371/ journal.pone.0244126. PMID: 33382764; PMCID: PMC7775097.

17. Keehner J, Horton LE, Pfeffer MA, Longhurst CA, Schooley RT, Currier JS, Abeles SR, Torriani FJ. SARS-CoV-2 Infection after Vaccination in Health Care Workers in California. N Engl J Med. 2021 May 6;384(18):1774-1775. doi: 10.1056/NEJMc2101927. Epub 2021 Mar 23. PMID: 33755376; PMCID: PMC8008750. 